\title{
The Second Opinion Dispatch- Evaluating Decisions Made by an Ambulance Dispatch Center
}

\author{
E. Carlström ${ }^{1,2,4,5}$, P. Rotter ${ }^{1}$, B. Asplén ${ }^{2}$, J. Thörnqvist ${ }^{2}$, P. Örninge ${ }^{2}$, M. Kihlgren ${ }^{2} \&$ A. Khorram-Manesh ${ }^{1,3}$ \\ ${ }^{1}$ Unit of Security and Preparedness, Gothenburg, Sweden \\ ${ }^{2}$ Unit of Prehospital Conduction, Gothenburg, Sweden \\ 3 Department of Surgery, Institute of Clinical Sciences, Sahlgrenska Academy, Gothenburg University, \\ Gothenburg, Sweden \\ ${ }^{4}$ Institute of Health and Care Sciences, Sahlgrenska Academy, Gothenburg University, Gothenburg, Sweden \\ ${ }^{5}$ University College of Southeast Norway, Borre, Norway \\ Correspondence: Eric Carlström Professor, Ph.D., ESB, Regionens Hus, 40544 Gothenburg, Sweden. Tel: \\ 46-702-738-126. E-mail: eric.carlstrom@gu.se
}

Received: September 24, 2017 Accepted: October 20, 2017 Online Published: October 24, 2017

doi:10.5539/gjhs.v9n12p107

URL: https://doi.org/10.5539/gjhs.v9n12p107

\begin{abstract}
Background: The efficacy and precision of dispatching right ambulance to right patient demands a proper decision-making. Wrong decisions may lead to overloading of emergency departments and shortage of ambulances. The aim of this study was to evaluate and improve the process of prehospital resource allocation conducted by a Swedish ambulance dispatch center.

Methods: A team of three experienced ambulance and dispatch nurses evaluated the decisions made by the dispatch center. The method chosen was "Action Research" divided into five actions during 76 days. In the first action, the team listened passively to the calls. The team gradually increased its involvement in the process of decision-making during the actions.

Results: During the actions, specific keywords indicating a need for evaluation were identified. The results showed a need to change the primary decisions in 486 cases out of 24,800 calls $(2 \%)$. The most common measure after an evaluation was to change an ordinary ambulance transportation to an assessment vehicle staffed by a nurse or a physician who would select an appropriate care level (hospital vs. primary healthcare).
\end{abstract}

Conclusion: This model not only optimized the prehospital resources but also changed the process of decision-making at the dispatch center and improved their staffs' ability to optimize the allocation of emergency resources.

Keywords: ambulance dispatch center, resource allocation, prehospital care, action research

\section{Introduction}

Globally, overcrowding at emergency departments and ambulance shortage is an everyday challenge (Claesson et al., 2012; Crilly et al., 2014), which necessitates a proper and appropriate utilization of existing resources within healthcare (Peyravi et al., 2015).

The challenge to make correct decisions at the Ambulance Dispatch Center (ADC) in order to avoid over- and under-triage is well-known. Earlier studies have shown a discrepancy in the decision-making between ADC and ambulance crews on site, with regard to patients' prioritization and type of transport needed (Khorram-Manesh et al., 2011). The former over utilizes the available resources, while the latter endangers patients' safety. In an Italian study, the nursing dispatch performance in detecting high-acuity patients in need of physician interventions revealed that ADC nurses without field experience (ambulance work) were not able to correctly identify the need for a physician on the scene. Consequently, their decisions resulted in an over-triage and over-utilization of available physicians (Leopardi \& Sommacampagna, 2013).

Almost all dispatch centers have a medical index by which the ADC staff use an algorithm to triage patients' calls in different categories and dispatch an appropriate means of transportation. In a Swedish context, studies have 
shown that the medical index used in Sweden results in up to $20-25 \%$ over-triage and very low percent of under-triage in trauma cases (Khorram-Manesh et al., 2011; Schagerlind et al., 2013). In a cross-sectional study from Norwegian ADCs, all registered medical emergency calls during a 72 hour period could demonstrate that although the official Norwegian policy is to always use a national index for decision-making, it was practiced in only $75 \%$ of the calls (Ellensen et al., 2014). The main reason for not using the index was a lack of the necessary keywords in order to properly assess acute medical emergency situations. In another study, the sensitivity and specificity of the priority grading made by the staff in a regional Swedish ADC were measured. The results showed a high sensitivity but low specificity in the dispatch system. Most of the patients with a high and acute need of an ambulance were correctly identified, but many patients without that need were also given a high priority ambulance service (Ek et al., 2013).

An interview study of ADC staff revealed that the decisions to dispatch ambulances were both challenging and difficult. Difficulties included conveying medical advice without seeing the patient, teaching cardio-pulmonary resuscitation via telephone and dealing with intoxicated and aggressive callers. Conflicts with colleagues and ambulance crews as well as fear of making the wrong decisions were also mentioned. The authors suggested that recruiting experienced triage nurses to operate calls could contribute to an improvement in the work at the ADC. They also identified a need to improve the internal support at ADCs and have a blame-free culture in order to attract and retain employees (Ek \& Svedlund, 2015). In another study, long shifts and high workload were proven to prolong the call duration, reduce ADC staffs' performance ability and specificity of decisions they made. Consequently, they oversimplified decisions and promoted a repetitive behavior. Such a behavior can be characterized by standardization, i.e., a monotonous dispatch of a single type of resource, e.g., ambulances, in most cases based on a premature analysis. The authors suggested that the efficiency could be improved by work shift configurations at the ADC (Montassier et al., 2014). The ability to give a second opinion on the scene (evaluation of the ADC decision) was studied in an Iranian context. The results indicated that in $71.2 \%$ of the 879 cases, the recipients made the decision to not be transferred to the hospital after the ambulance staff's individualized advice and a brief assessment. In most cases, the symptoms had subsided before the ambulance staff arrived or during their stay. In $28.8 \%$, the ambulance staff discharged the patients from the scene or left them at their home after definitive treatment or advice. A primary diagnosis was made, and recommendations such as self-care guidance and planned visits to a health care center were given (Peyravi et al., 2015).

In western Sweden, where the data for this study was collected, the work and efficacy of the dispatch center has been examined (Khorram-Manesh et al., 2011), but alternative resources such as units of mobile prehospital assessment nurses, mobile prehospital physician's, and single responders have also been added to the ambulance resources during the last few years. However, there is no indication that these prehospital units have been utilized as planned since ADC staffs have had some difficulties in identifying appropriate patients in need of these alternative resources (Carlström \& Fredén, 2016).

The aim of this study was to evaluate and improve the process of prehospital resource allocation conducted by a Swedish ADC by using a second opinion dispatch team (SODT). We also aimed to evaluate the impact it may have on the process of decision-making at the ADC by improving the staffs' ability to allocate the right resources to the right patients at the right level of care.

\section{Method and Materials}

The Swedish ADC is part of a public multi-owner national company, which receives all emergency calls in the entire country. The ADC in western Sweden is situated in Gothenburg and has 89 employed operators, who use a medical index (since 1990s) in order to handle around 2,147,698 incoming calls every year.

\subsection{Procedures}

The SODT team responsible for an evaluation of the made decisions consisted of three nurses with experience from ambulance services as well as ADCs activities. They were equipped with online computers and communication tools in order to get a full real-time insight into the procedures, calls, and decisions made by the ADC-operators. They could also communicate with the ADC-operators. Consequently, they carried out a second opinion of the incoming calls. All decisions made by the ADC staff were evaluated, and potential cases for improvement were identified and alternative measures proposed. All ADC staff were informed and consented to participate in this research project. The study was approved as a quality control measure and did not require any ethical approval. No information regarding individual patients was registered.

The process was designed as an action research study (Whyte, 1991) and consisted of five different actions/stages during daytime from Monday to Friday (08.00-22.00) and Saturday to Sunday (10.00-19.00) for 76 days. The 
construction of the five actions was based on the accumulation of knowledge from action to action. Whenever there was a need to take a turn, i.e., focus on specific, not yet examined information in the calls or alert decisions, a new action was initiated (McNiff, 2013). The first two actions were purely fictive, testing dimensions, items, and depth of analysis in the calls (Sefton \& Windle, 2011). The third and fourth actions were partly fictive and partly sharp interactions with the responding operators at the ADC. The fifth action was sharp, interacting with the activities of the ADC and suggesting alternatives to the chosen resource allocated by the ADC staff (Whyte, 1991). In simple text, stage one was characterized by a passive follow-up of each call. Notes and new ideas and any new suggestions could be collected and discussed with the researcher after every action (Tillyer et al., 2014). New actions focusing on new or different dimensions and items in the data collection were chosen. Every fulfilled action was also analyzed and discussed. Change of actions was based on consensus between the team and the researchers (McNiff, 2013). The methods and data collection during the different actions were fixed in order to secure a stringent process for the collection of the data (Whyte, 1991). The information gained was shared with the ADC staff. The level of involvement from the opinion team increased after each step, and with every step the retroactive discussion decreased in favor of real-time discussions with ADC staff; at the final stage, the SODT was interactively involved in the decision process.

\section{Results}

A total of $486(2 \%)$ calls out of all $(n=24,800)$ were evaluated by the SODT during a period of 76 days. During the fifth action (A.5), a total of $222(2.3 \%)$ calls $(n=9,500)$ were evaluated during a 30 day long action period. The cases were triaged by the dispatch center in three levels, from one, i.e., a high degree of acuity to three, a low degree of acuity. The criteria for potential cases in need of evaluation were collected and adjusted from action to action, and eventually the inclusions criteria were established during the last three actions (A.3-A.5). Focusing on words and sentences in the written information from the alerts, the SODT discovered that the written information could mirror an ambiguity in the decision made by ADC staff. The most common outcome of such ambiguity was awarding a lower priority than expected to a specific preliminary diagnosis (A.5=145), e.g., a preliminary diagnosis of chest pain was prioritized as middle level on a three-level scale. Such ambiguity was then recorded using special expressions/terms such as "unclear information" ( $\mathrm{n}=91$ cases of all included alerts in A.5). Other frequent expressions (>10 times), were "not severe," "injured but no trauma," "prolonged disease course," "diffuse or diminishing symptoms," and "no pain" (Table 1).

Table 1. Distribution of expressions/terms used in the inclusion criteria to evaluate the decisions in A.5 ( $\mathrm{n}=222)$.

\begin{tabular}{ll}
\hline Lower priority than expected & 145 \\
Unclear information & 35 \\
Not severe & $>10$ \\
Injured but no trauma & $>10$ \\
Diffuse or diminishing symptoms & $>10$ \\
Prolonged disease course & $>10$ \\
No pain & $>10$ \\
Diminishing symptoms & $>5$ \\
Young person, No disease history & $>5$ \\
Undergoing control/follow-up & $>5$ \\
Not able to be transported in another vehicle & $>5$ \\
A suspicion of & $>5$ \\
Able to walk & $>5$ \\
Known disease & $>5$ \\
Assessed within the last few days & $>1$ \\
Multiple calls & $>5$ \\
No general symptoms & $>1$
\end{tabular}


The main reason for evaluating a decision was the possibility of using more appropriate alternative resources than an ambulance such as single responder, prehospital physicians, and assessment nurses, etc. In some cases, the patients could be treated on site, instead of being transported to the hospital (16 cases in A.5). Other reasons for an evaluation of a decision made by the ADC staff were the possibility of advising a patient to use other means of transportation than an ambulance to a primary healthcare center or to the closest emergency department as a low priority case (16 cases in A.5). The most common diagnoses/symptoms evaluated by the team were abdominal/urinary conditions, chest pain, and psychiatric conditions, followed by dyspnea and headache/vertigo. As much as $60 \%$ of the assessed abdominal/urinary diagnoses were urinary catheter-issues and urinary infections. More than half of the cases assessed as chest pain had no pain at all during the emergency call, but symptoms such as tachycardia, arrhythmia, or discomfort in the chest. As much as $20 \%$ of the psychiatric diagnoses were panic disorders, and more than $30 \%$ included plans or attempts to commit suicide. Less frequent diagnoses were lumbago, hemorrhage, concussion, and minor traumas (Table 2).

Table 2. Distribution of the most common $(>12)$ preliminary diagnosis of re-evaluated calls in all actions

\begin{tabular}{|c|c|c|c|c|c|c|}
\hline \multirow{2}{*}{ Preliminary diagnosis assessed by ACD-operators } & \multicolumn{5}{|c|}{ Actions } & \multirow[b]{2}{*}{ All } \\
\hline & A.1 & A.2 & A.3 & A.4 & A.5 & \\
\hline Abdominal/urinary & 5 & 6 & 49 & 7 & 31 & 98 \\
\hline Chest pain & 2 & 3 & 33 & 7 & 36 & 81 \\
\hline Psychiatry & 3 & 5 & 31 & 7 & 30 & 76 \\
\hline Dyspnea & 2 & 5 & 33 & 6 & 19 & 65 \\
\hline Headache/ vertigo & 3 & 3 & 30 & 1 & 16 & 53 \\
\hline Lumbago & 1 & 3 & 11 & 1 & 7 & 23 \\
\hline Haemorrhage & 2 & 1 & 11 & 2 & 5 & 21 \\
\hline Minor trauma & & 1 & 11 & 4 & 4 & 20 \\
\hline Commotio & 2 & 2 & 5 & 2 & 10 & 21 \\
\hline Fever & & 1 & 7 & 4 & 4 & 16 \\
\hline Allergy & & 1 & 4 & 2 & 5 & 12 \\
\hline Unclear/ other & 25 & 12 & 65 & 32 & 55 & 189 \\
\hline All & 45 & 43 & 290 & 75 & 222 & 486 \\
\hline
\end{tabular}

The most frequent resources suggested by the SODT in A.5 $(\mathrm{n}=222)$ were the mobile assessment nurse $(\mathrm{n}=129)$, single responders $(n=41)$, and prehospital physician $(n=38)$. The physicians were dispatched to the site in special cases e.g., children, cancer patients, etc. (Haner et al., 2015). An ambulance helicopter was used in 14 cases. Temporary lack of alternative resources limited the possibilities to re-dispatch the cases. The mobile assessment nurse and the physicians were located in the densest populated urban area in the region and single responders on three or as most four locations in the rural parts of the region during daytime. In A.5, the communication between the ADC-operators and the SODT were more frequent than in the earlier actions, and ADC operators were more receptive to the suggestions from the SODT. The whole project was perceived to be successful as a learning process in order to optimize the allocation of prehospital resources by the members of SODT.

\section{Discussion}

The aim of this study was to evaluate and improve the process of prehospital resource allocation conducted by a Swedish ambulance dispatch center by using a second opinion dispatch team (SODT). We also aimed to evaluate the impact it may have on the process of decision-making at the ADC by improving the staffs' ability to allocate the right resources to the right patients at the right level of care.

This study shows that the accuracy of the ADC operators in dispatching the right prehospital resources to the right patients, using the current medical index was high. The few percentage of the evaluated cases were due to the ambiguity in the perception of the medical conditions through the telephone lines, which in most of the cases resulted in the substitution of an ambulance with an assessment vehicle or a single responder. The use of an SODT model could show an improvement in the willingness of the ADC staff to optimize the use of other prehospital 
resources and consequently an improvement of the specificity of prehospital care. Finally, the model showed a perceived improvement of the ADC staffs triage and decision-making ability.

In several Swedish regions, the county councils are responsible for the ambulances, while the ADC is responsible for managing the use of them. This relationship has been pointed out as one reason behind the lack of optimization of ambulances and prehospital emergency resources (Carlström \& Fredén, 2016). Accordingly, the idea of an SODT was born. Even if only a few cases are in need of evaluation $(2.2 \%$ in A.5) in this study, the loss of ambulance resources is relatively substantial. Since the average length of an ambulance mission in one selective area of western Sweden is calculated to be two hours (Carlström \& Fredén, 2016), the 222 evaluable cases found in the fifth 30 day action of our study corresponds to at least 444 hours of improved availability of ambulance time. The relatively few over-triaged cases will also consume some of the prehospital and hospital resources. Thus, the process of evaluation may lead to a more cost-effective distribution of resources to better select patients in need. Furthermore, the use of other prehospital resources, in line with earlier research, will offer the right service to the right patients at the right time (Haner et al., 2015).

Our results also suggest that the combination of field experience and working activity at ADC will improve the individual operator's triage competence and ability to make decisions. Assessment of patients through a telephone call is difficult (Peyravi et al., 2015); thus, staff experienced in on scene situations can potentially make a more precise assessment than staff solely experienced from ADC (Ek \& Svedlund, 2015). This on-scene knowledge and experience, together with medical index and guidelines, will increase the safety of decisions made for the patients. The fact that most of the evaluations were found among broad and unspecific preliminary diagnoses such as abdominal conditions, chest pain, and dyspnea, etc. may indicate a need for including more specific keywords to the prehospital protocols or creating disease-specific protocols. In contrast to traumas and fractures, such keywords might be difficult to interpret since they mirror everything from unspecific symptoms to severe and life-threatening conditions (Ellensen et al., 2014). On the other hand, expressions such as "diminishing symptoms" or "not severe," etc. indicate the operator's ambiguity in using a protocol based on a high degree of standardization. Such a protocol has a tendency to suggest one type of condition that is appropriate for the use of one type of resource, even if the operators might be unsure about their decisions. The keyword standardization may also induce a repetitive behavior, i.e., dispatch ambulances instead of considering alternatives, a phenomenon which may also be seen during high workload and stressful situations, inducing simplified standardizations (Montassier et al., 2014).

Studies of the dispatch performance are often focused on the inclusion of life-threatening high acuity cases (Leopardia \& Sommacampagnaa, 2013). In contrast to those studies, this study was focused on patients who were not in need of immediate ambulance resources. Dispatching ambulances to these cases, irrespective of the cause, decreases their availability. In such a situation, the re-distribution of ambulances is of great interest. An SODT makes it possible to re-evaluate the decisions made immediately, and by using other available resources, it contributes to a fair distribution of the right resources to the right patients and at the right level of care. It is important, however, to realize that such teams may also be a threat to the ADC operators since they could nourish a culture of blame and feeling of scapegoating (Ek \& Svedlund, 2015). In order to prevent such a toxic environment, thus, it is necessary to implement the system by using step-wise stages as conducted in our research. The actions in each stage were made transparent, and the project was allowed to develop slowly during discussions with the ADC staff. That is why the most operative part of the project, i.e., decisions made by the SODT, was conducted at the last action stage (A.5). During the progressive stages of this study, the willingness of ADC operator to collaborate increased, and the initial discussions were transferred for mutual consultation, with a focus on patients' safety and adequate use of available prehospital resources.

\subsection{Limitations}

One study limitation is the Hawthorne effects that arise with action research. According to the SODT, the ADC operators changed their behavior during the project and became more positive and receptive to the existence of the SODT. At the end of the study period, ADC staff were willing to change their decisions according to the suggestions from the SODT.

Furthermore, SODT could also notice an improvement in the ADC operators' accuracy in assessment, triage, and dispatching during the project. Such an impact on the study object can bias the figures presented in the results. However, based on a pragmatic perspective, the impact of the research project on the ADC operators is an outcome itself and probably contributes to a changed behavior when assessing calls and dispatching ambulances.

Another limitation was the challenge faced by the second opinion team in order to choose the inclusion criteria for an evaluation of the decisions made. Such selection is very individual and may be biased by a team consisting of 
three members.

Finally, the outcome for the patients, whether evaluated or not was not investigated in this study. The final diagnosis related to the type of transport used is an important parameter that needs to be studied further.

\subsection{Conclusion}

Only $2 \%$ of decisions made by dispatch operators were in need of evaluation in a Swedish context. The reason for evaluation was the ambiguity in the perception of the medical condition, which in most of the cases resulted in the substitution of an ambulance with an assessment vehicle or a single responder. The use of a second dispatch model is one way to optimize prehospital resources, improve the specificity of prehospital care, and provide tailored care for the patient. It may also improve the ADC staffs triage and decision-making ability through a consultative and progressive process of learning.

\section{Ethical Approval}

NA.

\section{Competing Interests Statement}

The authors declare that there are no competing or potential conflicts of interest.

\section{References}

Carlström, E., \& Fredén, L. (2016). The First Single Responders in Sweden -Evaluation of a pre-hospital single staffed unit. Int Emerg Nurs., 32, 15-19. http://dx.doi.org/10.1016/j.ienj.2016.05.003

Claesson, A., Lindqvist, J., Ortenwall, P., \& Herlitz, J. (2012). Characteristics of lifesaving from drowning as reported by the Swedish Fire and Rescue Services 1996-2010. Resuscitation, 83(9), 1072-1077. https://doi.org/10.1016/j.resuscitation.2012.05.025

Crilly, J. L., Keijzers, G. B., Tippett, V. C., O’Dwyer, J. A., Wallis, M. C., Lind, J. F., ... \& Shiels, S. (2014). Expanding emergency department capacity: a multisite study. Australian Health Review, 38(3), 278-287. https://doi.org/10.1071/AH13085

Ek, B., Edström, P., Toutin, A., \& Svedlund, M. (2013). Reliability of a Swedish pre-hospital dispatch system in prioritizing patients. International emergency nursing, 21(2), 143-149. Retrieved from http://www.sciencedirect.com/science/article/pii/S1755599X11001200?via\%3Dihub

Ek, B., \& Svedlund, M. (2015). Registered nurses' experiences of their decision - making at an Emergency Medical Dispatch Centre. Journal of clinical nursing, 24(7-8), 1122-1131. https://doi.org/10.1016/j.ienj.2011.11.006

Ellensen, E. N., Hunskaar, S., Wisborg, T., \& Zakariassen, E. (2014). Variations in contact patterns and dispatch guideline adherence between Norwegian emergency medical communication centres-a cross-sectional study. Scandinavian journal of trauma, resuscitation and emergency medicine, 22(1), 2. http://dx.doi.org/10.1186/1757-7241-22-2

Haner, A., Örninge, P., \& Khorram-Manesh, A. (2015). The role of physician-staffed ambulances: the outcome of a pilot study. Journal of Acute Disease, 4(1), 63-67. https://doi.org/10.1016/S2221-6189(14)60086-X

Khorram-Manesh, A., Montán, K. L., Hedelin, A., Kihlgren, M., \& Örtenwall, P. (2011). Prehospital triage, discrepancy in priority-setting between emergency medical dispatch centre and ambulance crews. European Journal of Trauma and Emergency Surgery, 37(1), 73-78. https://doi.org/10.1007/s00068-010-0022-0

Leopardi, M., \& Sommacampagna, M. (2013). Emergency nursing staff dispatch: sensitivity and specificity in detecting prehospital need for physician interventions during ambulance transport in Rovigo Emergency Ambulance Service, Italy. Prehospital and disaster medicine, 28(5), 523-528. https://doi.org/10.1017/S1049023X13008790

McNiff, J. (2013). Action research: Principles and practice. Routledge.

Montassier, E., Labady, J., Andre, A., Potel, G., Berthier, F., Jenvrin, J., \& Penverne, Y. (2015). The effect of work shift configurations on emergency medical dispatch center response. Prehospital Emergency Care, 19(2), 254-259. http://dx.doi.org/10.3109/10903127.2014.959217

Peyravi, M., Örtenwall, P., \& Khorram-Manesh, A. (2015). Can Medical Decision-making at the Scene by EMS Staff Reduce the Number of Unnecessary Ambulance Transportations, but Still Be Safe?. PLoS currents, 7, 1-5. https://doi.org/10.1371/currents.dis.f426e7108516af698c8debf18810aa0a 
Schagerlind, L., Örtenwall, P., Widgren, B. R., Taube, M., Asplén, B., Ominge, P., \& Khorram-Manesh, A. (2013). Validation of Swedish emergency medical index in trauma patients. J Emer DIsaster Med, 2(1), 1-6. Retrieved from http://www.jedm.org/index.php?journal=jedm\&page=article\&op=view\&path $\% 5 \mathrm{~B} \% 5 \mathrm{D}=19$

Sefton, T., \& Windle, S. (2011). Mediating the message of action research. Journal of Teaching and Learning, 7(2), $13-28$.

Tillyer, R., Tillyer, M. S., McCluskey, J., Cancino, J., Todaro, J., \& McKinnon, L. (2014). Researcher-practitioner partnerships and crime analysis: a case study in action research. Police Practice and Research, 15(5), 404-418. http://dx.doi.org/10.1080/15614263.2013.829321

Whyte, W. F. E. (1991). Participatory action research. Sage Publications, Inc.

\section{Copyrights}

Copyright for this article is retained by the author(s), with first publication rights granted to the journal.

This is an open-access article distributed under the terms and conditions of the Creative Commons Attribution license (http://creativecommons.org/licenses/by/4.0/). 